\title{
WHY DIELS-ALDER REACTIONS ARE NON-CONCERTED PROCESSES
}

\author{
LUIS R. DOMINGO
}

${ }^{1}$ Universidad de Valencia, Departamento de Química Orgánica, Dr. Moliner 50, E-46100 Burjassot, Valencia, Spain.

\begin{abstract}
Two exhaustive topological analyses using the Electron Localisation Function (ELF) along the non-polar Diels-Alder reactions of 1,3-butadiene [J. Phys. Chem. A 107, 6014 (2003)] and cyclopentadiene [Org. Biomol. Chem. 8, 5495, (2010)] with ethylene established that the bonding changes along these reactions are non-concerted. Herein, the ELF bonding changes along the intramolecular Diels-Alder reaction of 1-(hex-5-enyl)cyclohexa-1,3-diene is analysed. The geometrical restrictions imposed by the tether break the synchronicity in the single bond formation in this non-polar reaction. The present ELF topological analysis along this intramolecular Diels-Alder reaction supports the earlier findings that established the non-concerted nature of Diels-Alder reactions.
\end{abstract}

\section{INTRODUCTION}

In a recent manuscript devoted to the enzyme-catalyzed $[4+2]$ cycloaddition in the biosynthesis of spinosyn A, the authors stated that "The hallmark of Diels-Alder [4 + 2] cycloadditions is that they are concerted they proceed without forming any transient intermediates en route to the final product." This assertion is not correct because the definition of a Diels-Alder (DA) reaction, which is a reaction between a conjugated diene and an alkene to form a cyclohexene, should not include any mechanistic consideration. DA reactions can take place via a one-step mechanism or stepwise mechanisms. The inaccuracy in the use of the "concerted" word is taken to an extreme in an related article entitled "A Diels-Alderase at Last" in which the author establishes "The Diels-Alder reaction is the quintessential [4+2] cycloaddition. As illustrated in Scheme 1, in its simplest form buta-1,3-diene and ethylene react through a single pericyclic transition state (represented in brackets) to form cyclohexene. Two carbon-carbon $\pi$ bonds are lost, and two new carboncarbon $\pi$ bonds form simultaneously, an enthalpically favorable event. A key aspect of this process is the lack of charge development along the reaction coordinate, in keeping with the concerted, synchronous reorganization of six $\pi$ electrons". ${ }^{2}$ In this line, most computational chemists claim that DA reactions have a concerted mechanism.

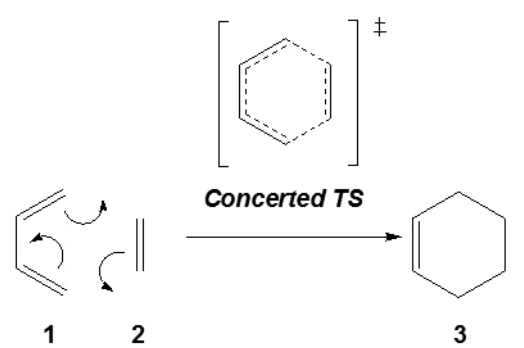

Scheme 1. Proposed pericyclic concerted mechanism for the DA reaction between butadiene 1 and ethylene 2 .

These mechanistic considerations come from the definition given in 1965 by R. B. Woodward and R. Hoffmann for pericyclic reactions ${ }^{3}$ as "reactions in which all first order changes in bonding relationship take place in concert on a close curve". ${ }^{4}$ The pericyclic model assumes that the six electrons involved in DA reactions, four of the diene and two of the alkene, move in a concerted fashion around the six carbon atoms (see the arrows in Scheme 1). But, is this very simple and accepted model the actual mechanism of DA reactions?

In 2009, we proposed the polar Diels-Alder (P-DA) mechanism ${ }^{5}$ that is followed by most experimental DA reactions. In the polar mechanism, the favourable electronic interactions that appear between the nucleophilic and the electrophilic species at the transition state structure (TS) of the reaction exert the main control of the activation energy of the reaction. The more polar a DA reaction is, the faster it can take place. The polar character of a DA reaction is measured by means of analysing the global electron density transfer (GEDT) that takes place from the nucleophilic species to the electrophilic one at the TS of the reaction. ${ }^{5}$ Most experimental DA reactions involving nonsymmetric electrophilic species take place through high asynchronous TSs associated to $a$ two-stage one-step mechanism, ${ }^{6}$ in which only one single bond is formed at the first stage of the reaction. Theoretical studies devoted to the bonding changes along polar reactions clearly suggest a rejection of the pericyclic mechanism for P-DA reactions. ${ }^{7}$ Only few unfavourable DA reactions follow a non-polar mechanism, being the most representative case the non-polar DA (N-DA) reaction between butadiene $\mathbf{1}$ and ethylene 2 . A question that remains is: are the bonding changes in this prototype of DA reaction concerted?

The first TS for the DA reaction between butadiene $\mathbf{1}$ and ethylene $\mathbf{2}$ was proposed by A. Wassermann in $1935 .{ }^{8}$ It was suggested that the lengths of the two forming bonds in the symmetric TS were $2.0 \AA$, a distance close to the 2.2 $\AA$ currently obtained (see Figure 1). ${ }^{9}$ Today, this synchronous TS is presented as a prototype for DA reactions in all textbooks.

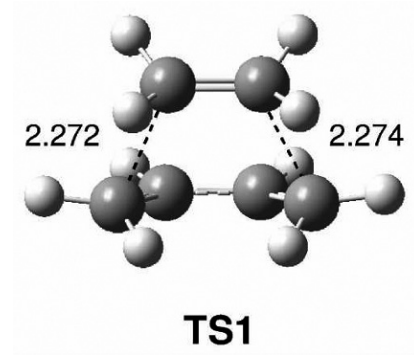

Figure 1. B3LYP/6-31G* transition structure TS1 associated with the DA reaction between butadiene $\mathbf{1}$ and ethylene $\mathbf{2}$.

Computational chemists describe a molecular mechanism through the characterization of stationary points involved in the reaction, that is, reagents, TSs, feasible intermediates and products. Usually, they characterise a molecular mechanism by an analysis of the TS involved in the reaction. Thus, for the DA reaction between butadiene $\mathbf{1}$ and ethylene $\mathbf{2}$ it is accepted that the TS is associated to a synchronous $\mathrm{C}-\mathrm{C}$ single bond formation process, with the lengths of the two forming bonds having identical values. ${ }^{9}$ However, this simple analysis does not give any information about the evolution of the breaking and forming of the double bonds along the reaction. Since the pericyclic reaction model considers changes of all single and double bonds involved in the reaction, the analysis of the synchronicity in the $\mathrm{C}-\mathrm{C}$ single bond formation at the TS is not sufficient to support the pericyclic model.

The development of the Bond Evolution Theory (BET), ${ }^{10}$ based on the electron localisation function ${ }^{11}$ (ELF) analysis along a reaction, has allowed to characterise the bonding changes along an elementary reaction, ${ }^{12}$ and thus to establish whether an organic reaction is a concerted process. Recent mechanistic studies devoted to the bonding changes along N-DA reactions, including the $\mathrm{N}-\mathrm{DA}$ reaction between butadiene $\mathbf{1}$ and ethylene $\mathbf{2}$, have indicated that they do not have a concerted breaking/forming bond process, ${ }^{13-15}$ thus refuting the pericyclic mechanism. ${ }^{15}$ 
In order to offer additional evidence of the non-concerted nature of N-DA reactions, the intramolecular Diels-Alder (IMDA) reaction of 1-(hex-5-enyl) cyclohexa-1,3-diene 4 is analysed hereafter (see Scheme 2).

\section{RESULTS AND DISCUSSION}

Just as the DA reaction between butadiene $\mathbf{1}$ and ethylene $\mathbf{2}$, this IMDA reaction takes place through an elementary step, thus presenting a one-step mechanism. Therefore, triene 4 , the only TS, TS2, and product 5 , were located and characterised (see Scheme 2). In this IMDA reaction, the GEDT that takes place from the cyclohexadiene fragment to the side-chain $\mathrm{C}=\mathrm{C}$ bond is $0.01 \mathrm{e} .{ }^{16}$ This unappreciable GEDT, which is closer to that found in the N-DA reaction between butadiene 1 and ethylene 2, 0.03e, accounts for the high activation energy found in this N-DA reaction, $31.7 \mathrm{kcal} / \mathrm{mol}$ (see Scheme 2).
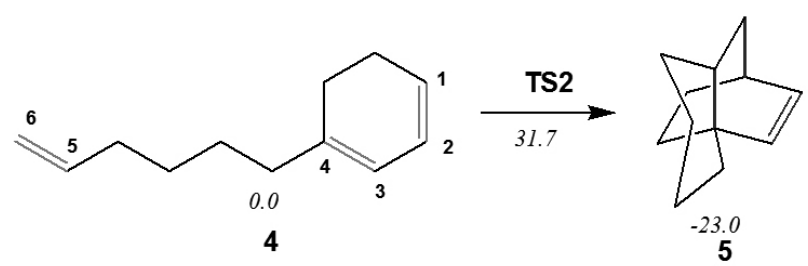

Scheme 2. IMDA reaction of 1-(hex-5-enyl)cyclohexa-1,3-diene 4 . Breaking bonds in red, and forming bonds in blue. Relative energies, in kcal/ mol, are given in italics.

The geometry of TS2 is given in Figure 2. Analysis of the distances between the carbons involved in the formation of the two new C-C single bonds, $\mathrm{d} 1=2.211 \AA$ and $\mathrm{d} 2=2.391 \AA$, shows a slight asynchronicity, $\Delta \mathrm{l}=0.18$, with respect to the symmetric TS1 found in the DA reaction between butadiene $\mathbf{1}$ and ethylene 2. This behaviour can be understood as a consequence of both the nonsymmetric substitution in the diene and ethylene fragment, and the presence of the tether in the intramolecular reaction. Note that TS3 associated with the intermolecular DA reaction between 1-methylcyclohexa-1,3-diene 6 and propene 7 is found slightly more advanced and more asynchronous than TS2. (see the geometry of TS3 in Note 17).

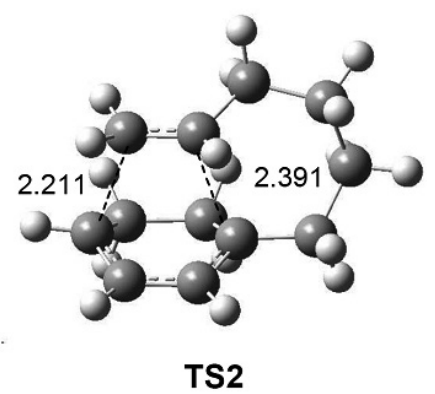

Figure 2. B3LYP/6-31G* transition structure TS2 associated with the IMDA reaction of 1-(hex-5-enyl)cyclohexa-1,3-diene 4.

As commented above, the single geometrical analysis performed at TS2 gives only information about the evolution of the formation of the two new $\mathrm{C}-\mathrm{C}$ single bonds. However, no information results about the breaking of the three double bonds present in triene 4 and the formation of the new $\mathrm{C} 2-\mathrm{C} 3$ double bond created in $\mathbf{5}$ is given, and consequently, the concerted nature of the reaction cannot be established.

DA reactions always involve three electron pairs belonging to the three double bonds present in reagents. Analysis of the bonding changes evolution demands a topological analysis of changes in electron density along the cycloaddition reaction. ${ }^{12}$ This analysis provides information about the breaking/ forming bond processes in an organic reaction.

After an analysis of the electron density, the ELF provides basins, that is, the domains in which the probability of finding an electron pair is maximal. ${ }^{11}$ Basins may be classified as core basins and valence basins. The latter are characterised by the synaptic order, i.e. the number of atomic valence shells in which they participate. Thus, there are monosynaptic, disynaptic, trisynaptic basins and so on. ${ }^{18}$ Monosynaptic basins, labelled V(A), correspond to the lone pairs or nonbonding regions, while disynaptic basins connect the core of two nuclei $\mathrm{A}$ and $\mathrm{B}$ and, thus, correspond to a bonding region between $\mathrm{A}$ and $\mathrm{B}$ and are labelled $\mathrm{V}(\mathrm{A}, \mathrm{B})$. Thus, while $\mathrm{C}-\mathrm{C}$ ' single bonds are characterised by the presence of one disynaptic basin $\mathrm{V}\left(\mathrm{C}, \mathrm{C}^{\prime}\right), \mathrm{C}-\mathrm{C}^{\prime}$ double bonds are charactesized by the presence of a pair of disynaptic basins, $\mathrm{V}\left(\mathrm{C}, \mathrm{C}^{\prime}\right)$ and $\mathrm{V}^{\prime}\left(\mathrm{C}, \mathrm{C}^{\prime}\right)$ (see ELF attractors of some simple molecules in Note 19). This description recovers the Lewis bonding model, therefore providing a very suggestive graphical representation of the molecular system.

Recently, we have shown that the CJ C single bond formation in both non-polar and polar organic reactions begins in the short CJ C distance range of $1.9-2.0 \AA$ by merging two monosynaptic basins, $\mathrm{V}(\mathrm{C})$ and $\mathrm{V}\left(\mathrm{C}^{\prime}\right)$, into a new disynaptic basin $\mathrm{V}\left(\mathrm{C}, \mathrm{C}^{\prime}\right)$ associated with the formation of the new CJ C' single bond. ${ }^{20,21}$ The $\mathrm{C}$ and $\mathrm{C}^{\prime}$ carbons characterised by the presence of the monosynaptic basins $\mathrm{V}(\mathrm{C})$ and $\mathrm{V}\left(\mathrm{C}^{\prime}\right)$ have been called pseudoradical centres. ${ }^{20}$ Accordingly, formation of a CJ C single bond can be understood to take place via a C-to-C pseudoradical coupling process in which each carbon furnishes the electron-density demanded for the formation of the new C-C single bond.

Recently, the seven phases characterising the bonding changes along the IRC of the DA reaction between butadiene 1 and ethylene $2^{13}$ have been categorised in four regions (see Figure 3) ${ }^{15}$ in the first one, A (in red colour), the three $\mathrm{C}-\mathrm{C}$ double bonds present in butadiene $\mathbf{1}$ and ethylene $\mathbf{2}$ break; this region comprises phases I to III; b) in the second region, B (in green colour), the formation of two pseudodiradical structures takes place by gathering electron density at the end carbons of the two unsaturated reagents, phases IV and V. The electron density demanded for the creation of the pseudoradical centres comes from the depopulation of the $\mathrm{C}-\mathrm{C}$ double bonds of the butadiene and ethylene moieties; $\mathrm{c}$ ) in the third region, $\mathrm{C}$ (in blue colour), which is constituted by the most relevant phase VI only, the formation of the two new C-C single bonds takes place through the C-to-C coupling between the pseudoradical centres generated in the previous phase $\mathrm{V}:{ }^{20}$ finally $\mathrm{d}$ ) in the fourth region, $\mathrm{D}$ (in violet colour), while the formation of the two C-C single bonds is completed, the formation of the new $\mathrm{C}=\mathrm{C}$ double bonds takes place. It is interesting to note that TS1 is located at the end of region $\mathrm{A}$
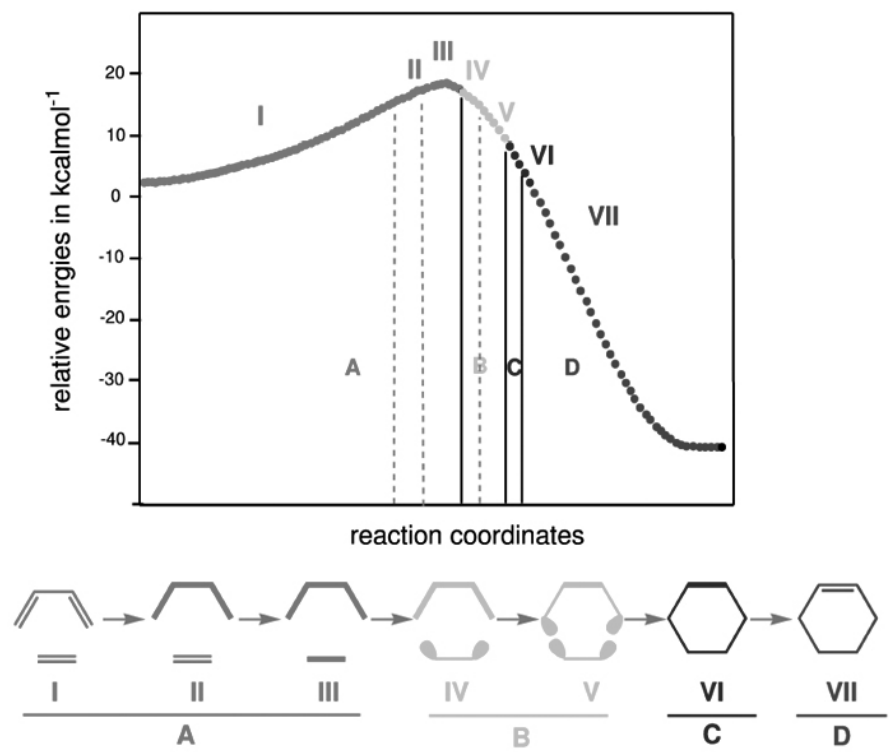

Figure 3. Reaction path calculated by means of the IRC method for the DA reaction between butadiene 1 and ethylene 2 . A bonding between atoms in all phases is demonstrated by the standard Lewis representation; however, in the case of phases IV and V, ellipses reflect the non-bonding electron density concentrated in the $\mathrm{C}$ atoms. The four colours represent the four regions in which the bonding changes can be categorised. 
In the present study, an ELF bonding analysis at the stationary points involved in the IMDA reaction of triene $\mathbf{4}$ is performed in order to establish the concerted or non-concerted nature of the DA reactions. In addition, the structure of the IRC IRC-12 $(\mathrm{d} 1=1.993 \AA$ and $\mathrm{d} 2=2.206 \AA)$ has been also analysed. The most relevant ELF attractors at these four structures are given in Figure 4.

ELF bonding analysis of triene 4 allows characterizing the three double bonds present in the Lewis structure of $4 ; \mathrm{C} 1-\mathrm{C} 2, \mathrm{C} 3-\mathrm{C} 4$ and $\mathrm{C} 5-\mathrm{C} 6$ (see 4 in Scheme 2). They are characterised by the presence of three pairs of disynaptic basins: $\mathrm{V}(\mathrm{C} 1, \mathrm{C} 2)$ and $\mathrm{V}^{\prime}(\mathrm{C} 1, \mathrm{C} 2), \mathrm{V}(\mathrm{C} 3, \mathrm{C} 4)$ and $\mathrm{V}^{\prime}(\mathrm{C} 3, \mathrm{C} 4)$, and $\mathrm{V}(\mathrm{C} 5, \mathrm{C} 6)$ and V'(C5,C6), integrating a total of 3.52e, 3.58e and 3.59e, respectively. ELF topological analysis of TS2 shows that it presents an electronic structure similar to that found in TS1. ${ }^{13}$ As was found in TS1, the three double bonds present in compound $\mathbf{4}$ have been already broken at TS2, while no monosynaptic basin, which will participate in the formation of the $\mathrm{C}-\mathrm{C}$ single bonds, appears at the four interacting carbons. At TS2 the disynaptic basins $\mathrm{V}(\mathrm{C} 1, \mathrm{C} 2), \mathrm{V}(\mathrm{C} 3, \mathrm{C} 4)$, and $\mathrm{V}(\mathrm{C} 5, \mathrm{C} 6)$ integrate $3.17 \mathrm{e}, 3.22 \mathrm{e}$ and $3.42 \mathrm{e}$, respectively. This electronic structure corresponds to that found at the end of region A in Figure 4. On the other hand, ELF analysis of product $\mathbf{5}$ shows that the two new C-C single bonds, characterised by the presence of two new disynaptic basins, $\mathrm{V}(\mathrm{C} 1, \mathrm{C} 6)$ and $\mathrm{V}(\mathrm{C} 4, \mathrm{C} 5)$, integrating $1.84 \mathrm{e}$ and $1.88 \mathrm{e}$, and the new $\mathrm{C} 3-\mathrm{C} 4$ double bond, characterised by the presence one pair of disynaptic basins, $\mathrm{V}(\mathrm{C} 2, \mathrm{C} 3)$ and $\mathrm{V}^{\prime}(\mathrm{C} 2, \mathrm{C} 3)$, integrating $3.55 \mathrm{e}$, are completely formed.

ELF analysis of point IRC-12 shows that while the formation of the C1-C6 bond is already in progress, see the presence of the disynaptic basin $\mathrm{V}(\mathrm{C} 1, \mathrm{C} 6)$ integrating $1.23 \mathrm{e}$ in IRC-12, the formation of the $\mathrm{C} 4-\mathrm{C} 5$ bond has not started yet. The ELF topological analysis of IRC-12 shows the presence of the two monosynaptic basins $\mathrm{V}(\mathrm{C} 4)$ and $\mathrm{V}(\mathrm{C} 5)$, integrating each one $0.55 \mathrm{e}$ and $0.48 \mathrm{e}$, responsible for the subsequent $\mathrm{C} 4-\mathrm{C} 5$ bond formation. This behaviour is in agreement with the observation that the $\mathrm{C}-\mathrm{C}$ single bond formation begins in the short C-C distance range of $1.9-2.0 \AA$. Note that the distance between the interacting carbons at IRC-12 are $\mathrm{d} 1=1.993 \AA$ and $\mathrm{d} 2=2.206 \AA$. Consequently, the low asynchronicity found in TS2 is sufficient to break the concerted C-C single-bond formation found in the symmetric TS1, but in both reactions, the breaking/forming bond processes that take place along these one-step reactions are non-concerted.

Finally, the ELF topological analysis of TS3 associated with the intermolecular DA reaction between 1-methylcyclohexa-1,3-diene 6 and propene 7 , which is more advanced and more asynchronous than intramolecular TS2, shows the presence of two monosynaptic basins, $\mathrm{V}(1)$ and $\mathrm{V}(6)$, integrating $0.36 \mathrm{e}$ and $0.33 \mathrm{e}$, respectively, prior to the formation of the $\mathrm{C} 1-\mathrm{C} 6$ single bond, and the absence of any monosynaptic basins at the interacting C4 and C5 carbons (see the ELF attractors of TS3 in Note 17). This picture is similar to that found in the asynchronous TS associated to the DA reaction between cyclopentadiene $\mathbf{8}$ and styrene $\mathbf{9 .}{ }^{14}$

Why are the bonding changes in DA reactions non-concerted?

As indicated above, we have shown that the $\mathrm{C}-\mathrm{C}$ single bond formation in both non-polar and polar organic reactions begins in the short C-C distance range of $1.9-2.0 \AA$ by coupling of two pseudoradical centres generated at the interacting carbons. In the N-DA reaction between butadiene $\mathbf{1}$ and ethylene 2, the two new C-C single bonds are created with an initial population of $c a$ $1.0 \mathrm{e}$ in the short region $\mathrm{C}$ by coupling of two pseudoradical centres, which gathered each one an electron population of $c a 0.5 \mathrm{e}$ at the end of region $\mathrm{B}$ (see Figure 3). ${ }^{13}$ The electron density found in the four pseudoradical centres comes from the depopulation of the three C-C double bonds present in both the diene and ethylene framework at the end of region A of the IRC. A similar pattern is found in the N-DA reaction between cyclopentadiene $\mathbf{8}$ and ethylene $\mathbf{2}^{14}$ Consequently, the electronic requirement demanded for the formation of the new $\mathrm{C}-\mathrm{C}$ single bonds means that the breaking of the three $\mathrm{C}-\mathrm{C}$ double bonds and the formation of the two $\mathrm{C}-\mathrm{C}$ single bonds will be non-concerted in these $\mathrm{N}-\mathrm{DA}$ reactions. Finally, note that the new C2-C3 double bond present in [4+2] cycloadducts is formed at the end of region $\mathrm{D}$.

Although the beginning and the end of the IMDA reaction of compound 4 is similar to that in the DA reaction between butadiene $\mathbf{1}$ and ethylene $\mathbf{2}$, both the nonsymmetric substitution on the diene and ethylene and the presence of the tether in the intramolecular reaction break the synchronicity (symmetry) in the formation of the two new C-C single bonds in this IMDA reaction. ELF bonding analysis of IRC-12 shows that at this point of the IRC the formation of the $\mathrm{C} 1-\mathrm{C} 6$ bond is already in progress, whereas the formation of the $\mathrm{C} 4-\mathrm{C} 5$ bond has not started yet. Note that the TS2 involved in the IMDA reaction of compound $\mathbf{4}$ is topologically similar to the TS1 involved in the N-DA reaction between butadiene $\mathbf{1}$ and ethylene $\mathbf{2}$. Consequently, the break of the synchronicity in the formation of the two new $\mathrm{C}-\mathrm{C}$ single bonds cannot be attained by an alone analysis performed at the TS of the reaction.
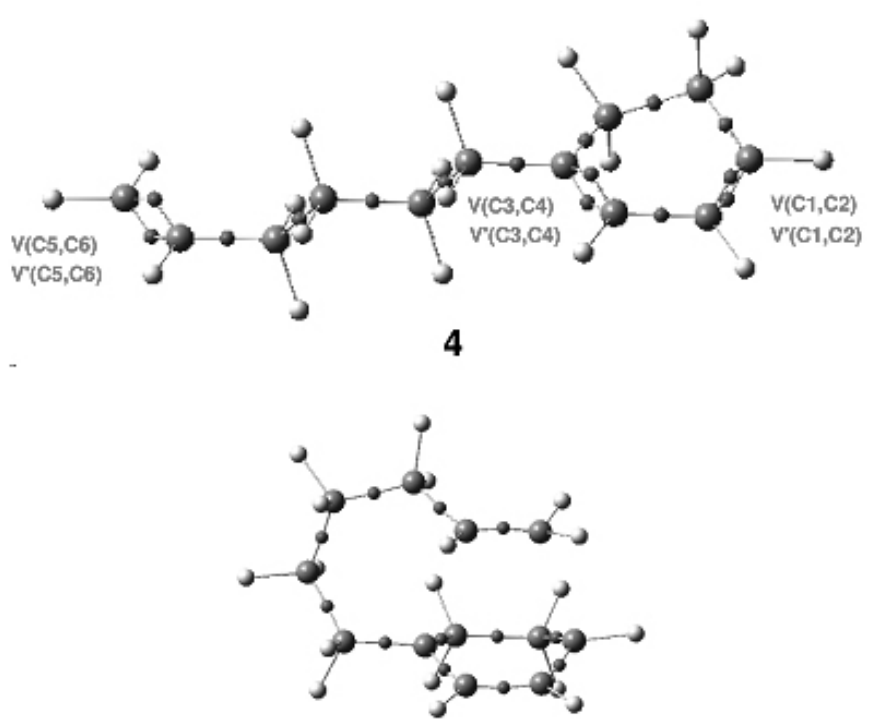

TS2

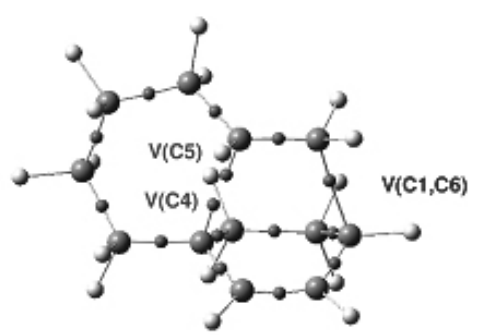

IRC-12

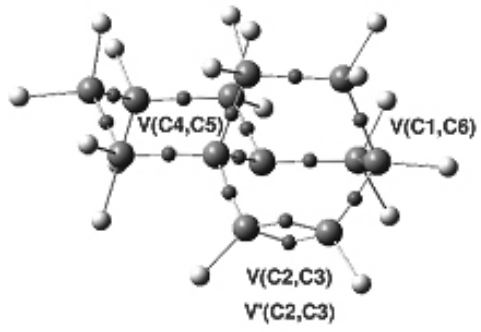

5

Figure 4. Most relevant ELF attractors at triene 4, TS2, IRC-12 and 5.

\section{CONCLUSIONS}

The bonding changes along the IMDA reaction of 1-(hex-5-enyl) cyclohexa-1,3-diene 4 have been studied by using an ELF topological analysis of the most relevant points along this intramolecular reaction at the B3LYP/6$31 \mathrm{G}^{*}$ computational level. The ELF topological analysis shows a closer break/ forming bond process than that found in the N-DA reactions of 1,3-butadiene $\mathbf{1}$ and cyclopentadiene $\mathbf{8}$ with ethylene $\mathbf{2}$. However, the geometrical restrictions imposed by the tether in this IMDA reaction break the synchronicity in 
the single bond formation in this non-polar reaction. In spite of the low asynchronicity found at the TS2 of this IMDA reaction, $\mathrm{Dl}=0.18$, the ELF topological analysis along the IRC indicates clearly that even the formation of the two new C-C single bonds are non-concerted. At the IRC-12 point, (d1 $=1.993 \AA$ and $\mathrm{d} 2=2.206 \AA$ ), while the formation of the C1-C6 single bond is already in progress, the formation of the $\mathrm{C} 4-\mathrm{C} 5$ bond has not started yet. The break of synchronicity found in the IMDA reaction $\mathbf{4}$ is enhanced in the N-DA reaction between 1-methylcyclohexa-1,3-diene 6 and propene $7 .{ }^{17}$

ELF bonding analysis along the reaction coordinates of N-DA reactions shows that the breaking of the three $\mathrm{C}-\mathrm{C}$ double bonds and the formation of the two $\mathrm{C}-\mathrm{C}$ single bonds and the new $\mathrm{C}-\mathrm{C}$ double bond are non-concerted processes. The electron density demanded for the formation of the two new $\mathrm{C}-\mathrm{C}$ single bonds must be reached before in the four interacting carbons by means of a previous depopulation of the three double bonds. This finding, which is only attained through a ELF topological analysis of the changes of electron density along a reaction, discards the Woodward and R. Hoffmann's proposal that the changes in electron density along a DA reaction are "in concert on a close curve". ${ }^{15}$

\section{Computational Methods}

DFT calculations were carried out using the B3LYP22 functional, together with the standard 6-31G* basis set. ${ }^{23}$ Optimisations were carried out using the Berny analytical gradient optimisation method. ${ }^{24}$ The stationary points were characterised by frequency computations in order to verify that TSs have one and only one imaginary frequency. The IRC ${ }^{25}$ paths were traced in order to check the energy profiles connecting each TS to the two associated minima of the proposed mechanism using the second order González-Schlegel integration method. ${ }^{26}$ The electronic structures of stationary points were analysed by the natural bond orbital (NBO) method ${ }^{27}$ and by the ELF topological analysis, $\eta(\mathbf{r}) .^{10}$ The ELF study was performed with the TopMod program ${ }^{28}$ using the corresponding monodeterminantal wavefunctions of the selected structures of the IRC. All calculations were carried out with the Gaussian 09 suite of programs..$^{29}$

\section{REFERENCES AND NOTES}

1 B. A. Hess, L. Smentek, Org. Biomol. Chem. 10, 7503, (2012).

2 C. A. Townsend, Chem. Bio. Chem. 12, 2267, (2011).

3 R. B. Woodward, R. Hoffmann, Angew. Chem., Int. Ed. Engl. 8, 781, (1969).

4 K. N. Houk, J. Gonzalez, Y. Li, Acc. Chem. Res. 28, 81, (1995).

5 L. R. Domingo, J. A. Sáez, Org. Biomol. Chem. 7, 3576, (2009).

6 L. R. Domingo, J. A. Saéz, R. J. Zaragozá, M. Arnó, J. Org. Chem. 73, $8791,(2008)$

7 (a) P. González-Navarrete, L. R. Domingo, J. Andrés, S. Berski, J. Comp. Chem. 33, 2400, (2012). (b) L. R. Domingo, P. Pérez, M. J. Aurell, J. Sáez, A. Curr. Org. Chem., 16, 2343, (2012). (c) L. Rhyman, P. Ramasami, J. A. Joule, J. A. Sáez, L. R. Domingo, RSC Adv. 3, 447, (2013).

8 A. Wasserman, J. Chem. Soc. 828, (1935).

9 E. Goldstein, B. Beno, K. N. Houk, J. Am. Chem. Soc. 118, 6036, (1996).

10 X. Krokidis, S. Noury, B. Silvi, J. Phys. Chem. A 101, 7277, (1997).

11 (a) A. Savin, A. D. Becke, J. Flad, R. Nesper, H. Preuss, H. G. von Schnering, Angew. Chem. Int. Ed. 30, 409, (1991). (b) B. Silvi, A. Savin, Nature 371, 683, (1994). (c) A. Savin, B. Silvi, F. Colonna, Can. J. Chem. 74, 1088, (1996). (d) A. Savin, R. Nesper, S. Wengert, T. F. Fassler, Angew. Chem., Int. Ed. Engl. 36, 1808, (1997).

12 V. Polo, J. Andrés, S. Berski, L. R. Domingo, B. Silvi, J. Phys. Chem. A, 112, 7128, (2008).

13 S. Berski, J. Andrés, B. Silvi, L. R. Domingo, J. Phys. Chem. A, 107, 6014, (2003).

14 L. R. Domingo, E. Chamorro, P. Pérez, Org. Biomol. Chem 8, 5495, (2010).

15 L. R. Domingo, Org. Chem. Curr, Res 2: 120. doi:10.4172/21610401.1000120 (2013).

16 J. Soto-Delgado, L. R. Domingo, R. Contreras, Org. Biomol. Chem. 8, $3678,(2010)$.

17 TS3 associated with the intermolecular DA reaction between 1-methylcyclohexa-1,3-diene 6 and propene 7 is found slightly more advanced and more asynchronous than TS2. $\Delta \mathrm{l}=0.38$, GEDT $=0.01 \mathrm{e}$.

(a) B3LYP/6-31G* geometry and (b) most relevant ELF attractors of TS3 associated with the DA reaction between 1-methylcyclohexa-1,3-diene 6 and propene 7.

18 B. Silvi, A. Savin, Nature 371, 683, (1994).
19 ELF attractors of some simple organic molecules

20 (a) L. R. Domingo, E. Chamorro, P. Pérez, Lett. Org. Chem. 7, 432, (2010). (b) L. R. Domingo, J. A. Sáez, J. Org. Chem. 76, 373, (2011).

21 (a) L. R. Domingo, P. Pérez, J. A. Sáez, Org. Biomol. Chem. 10, 3841, (2012). (b) L. R. Domingo, P. Pérez, J. A. Sáez, Tetrahedron, 69, 107, (2013). (c) L. R. Domingo, P. Pérez, J. A. Sáez, RSC Adv. 3, 7520, (2013).

22 (a) A. D. Becke, J. Chem. Phys. 98, 5648, (1993). (b) C. Lee, W. Yang, R. G. Parr, Phys. Rev. B 37, 785, (1988).

23 W. J. Hehre, L. Radom, P. v. R. Schleyer, J. A. Pople, in Ab initio Molecular Orbital Theory, Wiley, New York, 1986.

24 (a) H. B.Schlegel, J. Comput. Chem. 2, 214, (1982). (b) H. B. Schlegel, In Modern Electronic Structure Theory (Ed.: Yarkony, D. R.) World Scientific Publishing: Singapore, 1994.

25 K. J. Fukui, Phys. Chem. 74, 4161, (1970).

26 (a) C. González, H. B. Schlegel, J. Phys. Chem. 94, 5523, (1990). (b) C. González, H. B. Schlegel, J. Chem. Phys. 95, 5853, (1991).

27 (a) A. E. Reed, R. B. Weinstock, F. Weinhold, J. Chem. Phys. 83, 735, (1985). (b) A. E. Reed, L. A. Curtiss, F. Weinhold, Chem. Rev. 88, 899, (1988).

28 S. Noury, X. Krokidis, F. Fuster, B. Silvi, Comput. Chem. 23, 597, (1999).

39 M. J. Frisch, Gaussian 09 Gaussian, Inc., Wallingford CT, 2009.

\section{ACKNOWLEDGMENTS}

I want to thank to Fondecyt for continuous support to the investigation through Cooperación Internacional Fondecyt (grant No. 1100278). 